\title{
IMPLEMENTACIÓN DE UN SISTEMA DE INFORMACIÓN GEOGRÁFICO PARA LA ACTUALIZACIÓN DE DATOS DE USUARIOS DE AGUAS MEXICANAS UBICADOS EN LOS ACUÍFEROS DEL ESTADO DE SONORA, MÉXICO.
}

EPISTEMUS

ISSN: 2007-8196 (electrónico)

ISSN: 2007-4530 (impresa)

Gerardo Ramírez Uribe 1 Jesús Fernando García Arvizu 2 Jesús Quintana Pacheco ${ }^{3}$ Arturo Ojeda de la Cruz ${ }^{4}$ José Refugio Silvestre Ortiz ${ }^{5}$

Recibido: 14 de marzo de 2017 Aceptado: 30 de mayo de 2018

Autor de Correspondencia:

Dr. Gerardo Ramírez Uribe

Correo: guribe@industrial.uson.mx

\section{Resumen}

Este proyecto presenta el marco conceptual de una metodología sobre la implementación de un sistema de información geográfica (SIG) para la actualización de datos de usuarios de aguas mexicanas ubicados en los acuíferos del Estado de Sonora, México. Este SIG, se basa en el análisis del contenido para el proceso de actualización de datos de usuarios propietarios de aprovechamientos de recursos hídricos, con el fin de presentar algunos elementos relacionados a establecer ventajas competitivas en las organizaciones que necesiten mejorar la actualización de datos de usuarios mediante un SIG.

Las soluciones a los problemas relacionados con el agua dependen de muchos factores, entre los que se encuentran las instituciones que la administran, los procesos mediante los cuales se gestiona el agua, condiciones socio-políticas, el desarrollo y las prácticas de gestión y

Los marcos jurídicos existentes. En esta situación, las soluciones innovadoras, como la recarga de las aguas sub-terraneas, los cambios en la estructura de tarifas de agua, el análisis institucional y técnica de manejo de datos como SIG están ganando importancia.

Palabras claves: sistemas. Acuiferos información geográfica

\section{Abstract}

This project presents the conceptual framework of a methodology on the implementation of a geographic information system (GIS) for the updating of data of Mexican water users located in the aquifers of the State of Sonora, Mexico. This GIS is based on the analysis of the content for the process of updating data of users who own water resources, in order to present some elements related to establishing competitive advantages in organizations that need to improve the updating of user data through a GIS.

Solutions to water-related problems depend on many factors, including the institutions that manage water, the processes by which water is managed, sociopolitical conditions, development and management practices, and

Existing legal frameworks. In this situation, innovative solutions, such as subsurface water recharge, changes in water tariff structure, institutional analysis and data management techniques such as GIS are gaining importance.

Keywords: Systems, Aquiferos Geographical Information 


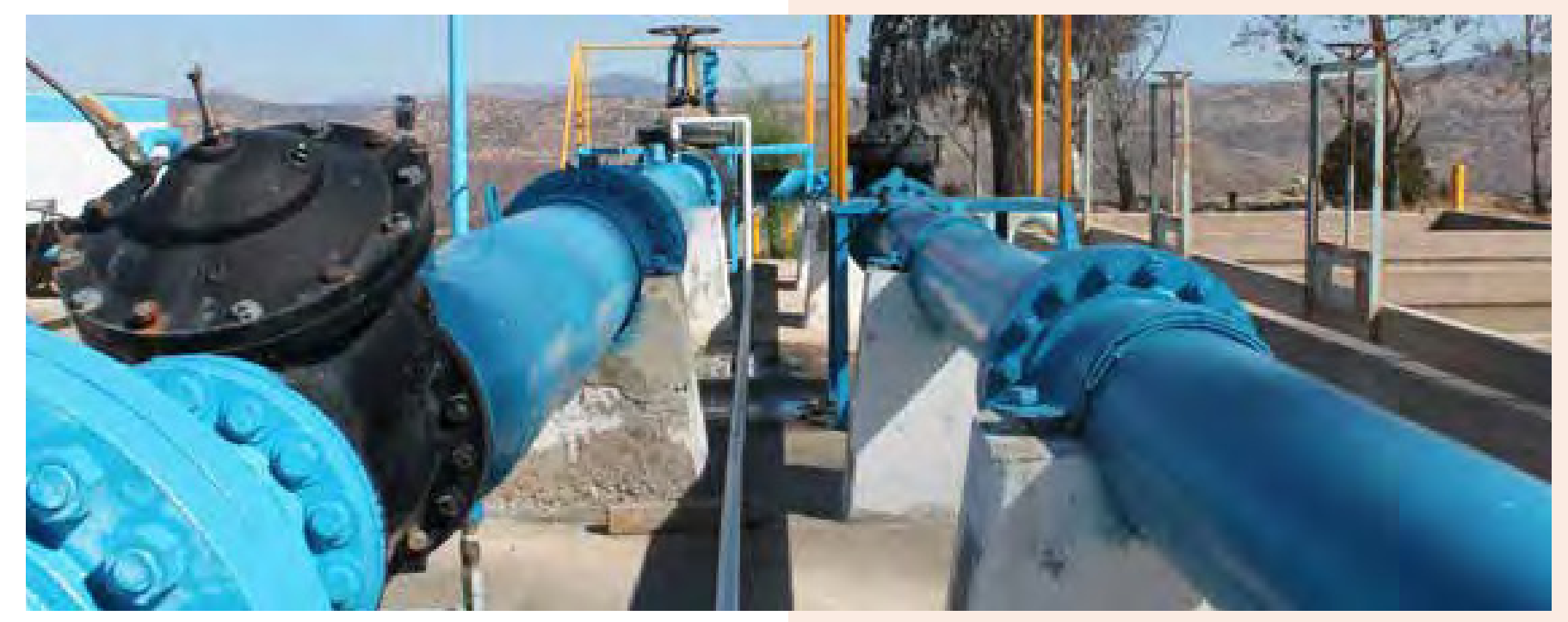

\subsection{Preparación de la Sistema de Información Geográfico (SIG).}

Es necesario primeramente presentar el proyecto ante las autoridades de la Comisión Nacional del Agua (CONAGUA) para recibir la aprobación por parte de ellos, se continúa con la conjunción de un grupo de trabajo que será responsable de la logística del desarrollo, estos mismos serán los responsables de recopilar, capturar la información con la cual se diseñara la SIG y para finalizar se aplicará una encuesta a los usuarios dueños de los aprovechamientos que forman parte de las cuencas. A continuación se explica a detalle cada una de las actividades antes mencionadas.

\subsubsection{Presentar el proyecto a la CONAGUA.}

El proyecto de desarrollar un SIG se presenta ante la CONAGUA, el gerente de inspección y medición de la subsecretaria general de administración de agua, Roberto Merino Carreón toma la decisión de aceptar o no el proyecto, y que este pueda ser implementado, así como también los ajustes necesarios para la aplicación y desarrollo del sistema.

\subsubsection{Conformar el grupo de trabajo para llevar a cabo la logística del desarrollo.}

Una vez que se ha formalizado el proyecto ante la CONAGUA se procede a conformar el grupo de trabajo para llevar a cabo la logística del desarrollo.

Estas personas serán responsables de la recopilación y análisis de la información existente de los aprovechamientos hídricos, la elaboración del programa de actividades de campo, concertación de reuniones de trabajo con los distintos órdenes de gobierno y organizaciones de usuarios, el levantamiento de las cédulas del censo de aprovechamientos hídricos subterráneos, la captura y procesamiento de cédulas de campo en la plataforma de sistemas de información Geográfica SIG.

\subsubsection{Diseño y aplicación de una encuesta para determinar el estado los aprovechamientos de las cuencas.}

La preparación del SIG empieza con una encuesta aplicada a los usuarios dueños de los aprovechamientos situados en las cuencas cuyos resultados presentan la orientación de las unidades afectadas en la organización. Los objetivos de la orientación son en primer lugar, determinar la situación legal y administrativa del aprovechamiento, corroborar las características del aprovechamiento e infraestructura instalada (equipo y bomba de extracción de agua), ubicación del aprovechamiento, etc. En la figura 3.2 se muestra parte de la encuesta.

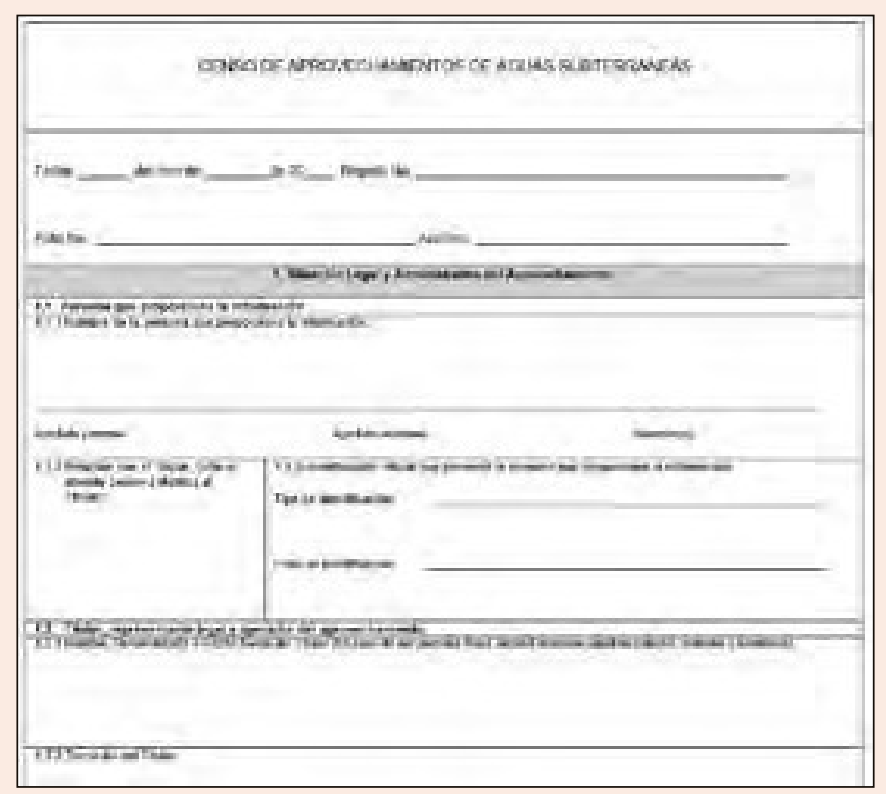

Figura 3.2 Encuesta para actualización de datos de usuarios dueños de los aprovechamientos de las cuencas. 


\subsection{Aplicación de la Encuesta}

\subsubsection{Recopilación y análisis de la información existente de los aprovechamientos hídricos.}

Se procederá a recopilar, analizar y geoposicionar las bases de datos del Registro Público de Derechos de Agua (REPDA) de los aprovechamientos subterráneos en estudio, finalmente estos datos se agregarán a la Plataforma Geográfica.

También se procederá a recopilar, procesar y analizar la información que se refiere a los censos de los aprovechamientos hídricos con que cuenta el Organismo de Cuenca, los organismos Operadores del Estado de sonora y Las Unidades Académicas locales.

\subsubsection{Reuniones de trabajo con los distintos órdenes de gobierno y organizaciones de usuarios.}

Se elaborará la agenda del día de las reuniones de trabajo y se levantará una minuta de trabajo con los resultados y acuerdos y se tomará la lista con los datos generales de los asistentes. En dichas reuniones, se expondrán el contenido y los alcances del presente estudio, así como la programación general y se presentaran ante el Coordinador Estatal y los responsables de las brigadas de campo y se solicitará el apoyo de los distintos órdenes de gobierno para realizar en tiempo, forma y calidad los trabajos encomendados.

\subsubsection{Elaboración del programa de actividades de campo.}

Se elaborará un programa detallado de trabajo de actividades de campo del acuífero en estudio, para ello se deberá utilizar la Plataforma Geográfica que se ha elaborado; en esta se deberá contemplar una capa que contenga una cuadricula con módulo de $1.0 \mathrm{~km}$ X $1.0 \mathrm{~km}$ y una clave por cada celda formada.

Los transectos nulos serán celdas que por sus características se asume que no contienen aprovechamientos por lo que se deberá considerar al menos la siguiente información para definirlos: accesibilidad a la zona, poblaciones cercanas, pendientes bruscas o abruptas atreves de curvas de nivel.

Que los perfiles geológicos indiquen zonas no apropiadas para contener agua. Sin embargo se deberá verificar las zonas para asegurar que no existen aprovechamientos.

En función de la densidad de los aprovechamientos Hídricos subterráneos se determinará el número de brigadas necesarias para realizar el censo, la cobertura geográfica de cada una de las brigadas, las rutas a seguir para optimizar los recursos y el tiempo, los rendimientos programados, las fajas de la cuadrícula se levantará por día, semana y mes, de la misma forma se nombrará un Coordinador Estatal, quien será responsable en campo y en gabinete de los trabajos efectuados por las brigadas.

Posteriormente se someterá el programa de trabajo a revisión y autorización por parte de los supervisores de CONAGUA que será presentado en Microsoft Office Project y contendrá los siguientes datos genéricos del proyecto: estado, acuífero, nombre del coordinador estatal, nombres de los responsables de las brigadas. La figura 3.3 muestra la programación de campo en el Microsoft Project.

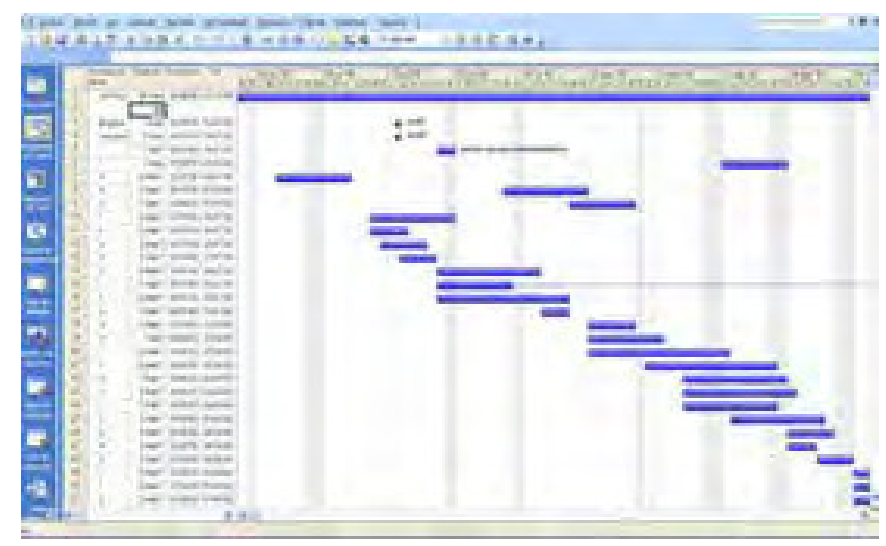

Figura 3.3. Programación de trabajos de campo

\subsubsection{Censo de aprovechamientos hídricos subterráneos.}

Finalizada la programación por acuífero y efectuadas las reuniones de trabajo con los distintos órdenes de gobierno y organizaciones de usuarios; se procede a realizar el censo de aprovechamientos subterráneos para lo cual se hará un barrido total pro las brigadas integradas para el acuífero en estudio, de cada una de las celdas de la matriz rectangular; en ese barrido se ubicarán , geoposicionarán, levantarán las cédulas de registro de campo de los aprovechamientos de acuerdo al formato de la encuesta y se tomarán fotografías digitales que ilustren las características de cada aprovechamiento.

Para determinar las coordenadas geográficas de casa aprovechamiento Hídrico que se localicen en cada celda, 
se utilizará un GPS de tipo Navegador con error máximo de $10 \mathrm{~m}$

Los brigadistas de campo levantarán las encuestas de registro de información de campo por cada uno de los aprovechamientos hídricos que localicen y geoposicionen en campo, para lo cual llenarán todos los campos de información que contiene la encuesta.

El código de identificación del aprovechamiento estará integrado por la clave del acuífero a cuatro dígitos, clave de la celda a seis dígitos (columna y renglón respectivamente sin coma), un guion bajo, la identificación de la brigada de campo (una letra) y el número progresivo del aprovechamiento por brigada a 3 dígitos, como se muestra en la tabla 3.1

\section{Tabla 3.1. Código de Identificación de los} aprovechamientos

\begin{tabular}{|c|c|}
\hline ACUÍFERO & NOMBRE DEL ACUIFERO \\
\hline CLAVE & CLAVE DEL ACUIFERO \\
\hline CLAVE DE LA CELDA & $\begin{array}{c}\text { NUMERO DE COLUMNA Y } \\
\text { RENGLON DE LA CUADRICULA }\end{array}$ \\
\hline BRIGADA & C \\
\hline $\begin{array}{c}\text { NUMERO PROGRESIVO } \\
\text { POR BRIGADA }\end{array}$ & 036 \\
\hline
\end{tabular}

\section{Quedando el folio como: 1916022001_C036}

Este código de aprovechamiento es importante que quede adecuadamente para evitar confusiones en la base de datos que se elaborará posteriormente,

El llenado de las encuestas de campo se realizará utilizando tinta color azul, con letra de molde legible y sin tachaduras, borraduras o enmendaduras que pongan en duda la captura de la información durante los trabajos de gabinete.

Durante el llenado en campo de la encuesta, se marcará cada aprovechamiento en el tren de descarga o en un punto visible próximo al mismo así como en el tablero de arranque, con una etiqueta autoadherible para exteriores media carta de poliéster para soportar el uso

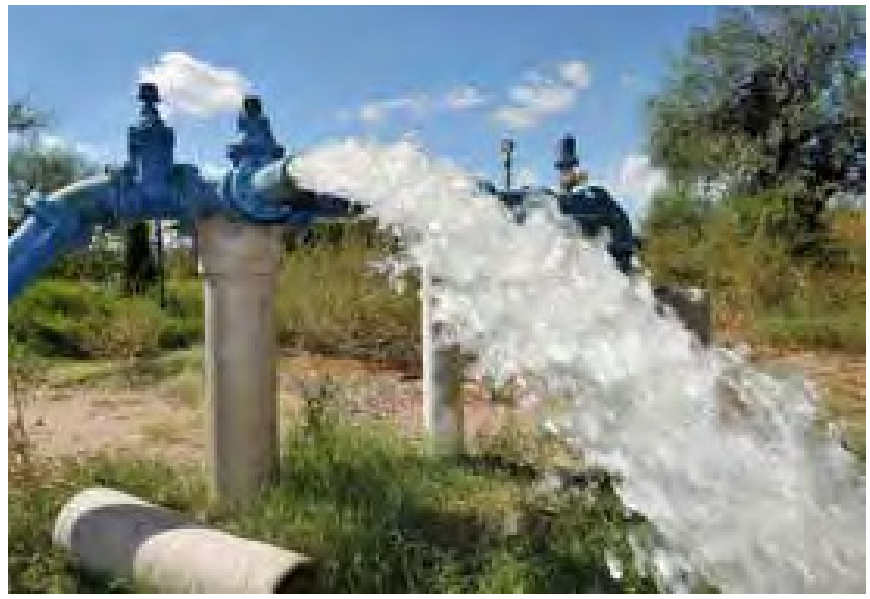

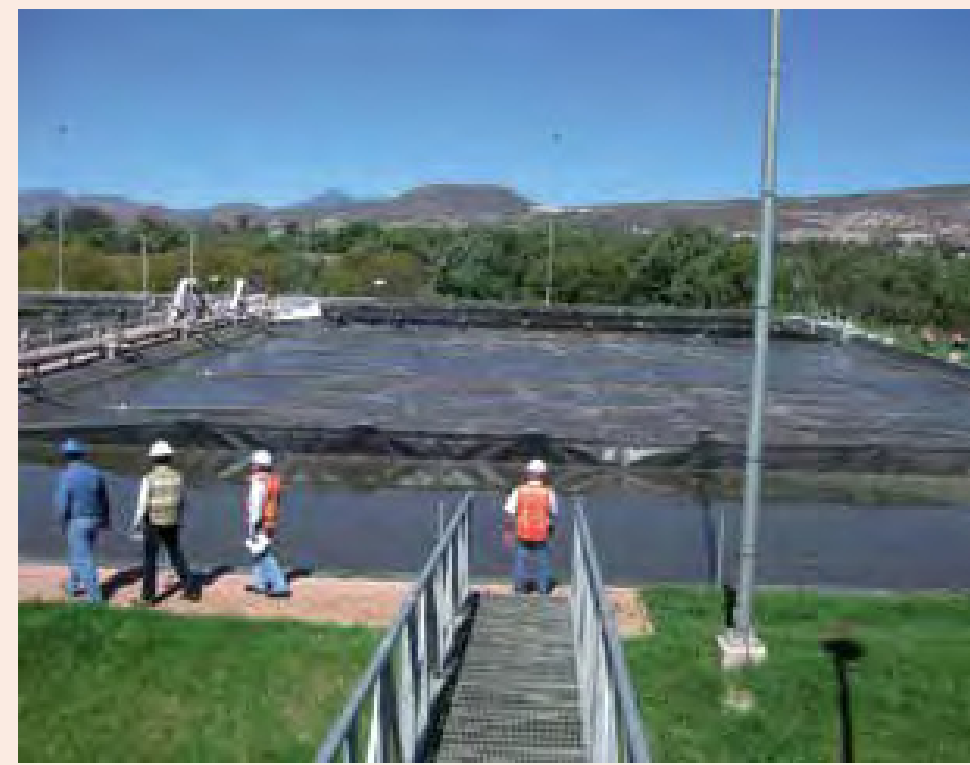

rudo, temperaturas extremas y humedad, en el centro el folio de aprovechamiento, seguido de la palabra censado y la fecha correspondiente, escritos con tinta indeleble o impresora laser.

Para garantizar la veracidad de los datos consignados en los campos de información de la cédula de registro de campo referentes al número de título, anexo y coordenadas geográficas en el título y que se trata del número de medidor y registro Permanente de Usuario (RPU) de la Comisión Federal de Electricidad (CFE), se le solicitará al usuario o persona que atiende a la brigada, muestre el original o la copia del título de concesión y el último recibo de consumo eléctrico asociado al aprovechamiento censado. Si al momento de la recopilación de datos el usuario no contara con la información solicitada la brigada deberá concertar una cita para la complementación del os datos faltantes.

Una vez Geoposicionado el aprovechamiento, las brigadas de campo, escribirán en un pintarrón de $60 \mathrm{x}$ $40 \mathrm{~cm}$ la fecha, el folio de la cédula de registro de campo, las coordenadas, en grados minutos y segundos y la denominación del aprovechamiento que le corresponda y se procederá a tomar una serie de al menos ocho fotografías del aprovechamiento, utilizando cámara digitales con una resolución mínima de 6 megapixeles. Las fotografías digitales que se tomarán deberán contener en el siguiente orden: cabezal y tren de descarga del aprovechamiento de aguas subterráneas, toma panorámica que incluya puntos de referencia del sitio, acercamiento de la lectura del medidor volumétrico (si existe), acercamiento de la lectura del medidor de corriente eléctrica de la CFE (si existe), folio o cédula del registro en el pintarrón de $60 \times 40 \mathrm{~cm}$ incluyendo tren de descarga de referencia, acercamiento a la caratula del Título de Concesión o Asignación en donde se observe el número de título de concesión y el nombre del titular al cual se asocia el aprovechamiento (si existe), 
acercamiento del anexo del título de concesión que corresponde al aprovechamiento censado, acercamiento al recibo de consumo eléctrico en donde se observe el RPU asignado por CFE.

Cada fotografía digital debe tener una resolución de $1.024 \times 768$ pixeles adecuando la toma a fin de evitar la duplicidad de datos, errores $u$ omisiones en la información recabada por cada una de las brigadas, se implementará mecanismos de control y validación que permitan garantizar la calidad de los trabajos de campo realizados antes de su integración en la plataforma Geográfica.

Para asegurar la confiabilidad El Coordinador Estatal proporcionará al Supervisor de CONAGUA las cédulas solicitadas, quien comprobará en campo la información recabada. En caso de encontrar error, omisión, falsedad de la información o que sea ilegible, el supervisor notificará Coordinador Estatal lo encontrado para que este verifique la totalidad de las cédulas levantadas por la brigada en cuestión.

\subsection{Diseño de la SIG}

La información recopilada de la encuesta que se refiere a los aprovechamientos hídricos será cargada en la SIG para la utilización en la realización del presente proyecto.

\subsubsection{Información contenida en la Plataforma Geográfica}

El sistema de Información Geográfica deberá contener: ortofoto a escala 1: 20000, los siguientes elementos cartográficos digitales a nivel estatal de: limites políticos de estados y municipios, acuíferos, límites de las Regiones hidrológicas, cuencas y Regiones hidrológicas, localidades a una escala de 1:50000, zonas de cultivo, almacenamientos (vasos Bordos Presas) 1:50000, ríos y Corrientes principales 1:50000, curvas de Nivel topográficas a 1:50000.

Se armará el proyecto de la plataforma Geográfica formado por un "DataFrame"; expresado en décimas de grado con los parámetros estándares ITRF92 (datum y esferoide GRS80) en donde se ubiquen geográficamente los acuíferos antes mencionados.

\subsubsection{Captura y procesamiento de cédulas de campo en la plataforma de sistemas de información Geográfico.}

Se integrará toda la información contenida en las encuestas, las fotografías de los aprovechamientos y la imagen digital de cada una de las cédulas levantadas, en una base de datos montada en el SIG. Dicha Plataforma deberá contener una capa para cada de los siguientes reportes: aprovechamiento s identificados con título de concesión o asignación, diferencias encontradas entre el título de Concesión o asignación y los datos de campo en los siguientes rubros: coordenadas geográficas, acuífero, titular del aprovechamiento y uso de las aguas nacionales, aprovechamientos identificados sin título de concesión o asignación y con algún trámite ante CONAGUA, aprovechamientos identificados sin título de concesión o asignación y sin ningún trámite ante CONAGUA, concesiones no encontradas durante el desarrollo de los trabajos.

Durante el desarrollo de los trabajos de campo y gabinete, se realizaran con representantes de CONAGUA reuniones para presentar los avances obtenidos, cuando lo solicite la CONAGUA y Contratista.

\section{RESULTADOS Y AVANCES DEL SISTEMA}

En esta sección, se muestran los resultados obtenidos de la aplicación de la metodología. En esta sección se verá el procesado de la encuesta, propuesta del software para el desarrollo, elaboración del SIG, captura de la información levantada en campo en el SIG.

\subsection{Procesado de la Encuesta}

Durante la ejecución del censo, las encuestas levantadas, son trasladadas al Departamento de Ingeniería Civil y Minas, para su validación, captura y procesamiento. El traslado se realiza semanalmente.

La captura de las encuestas se realiza en una base de datos previamente diseñada por especialistas en el programa Microsoft Access. La figura 4.1 muestra la interface de Access para agregar información de la encuesta en la base de datos.

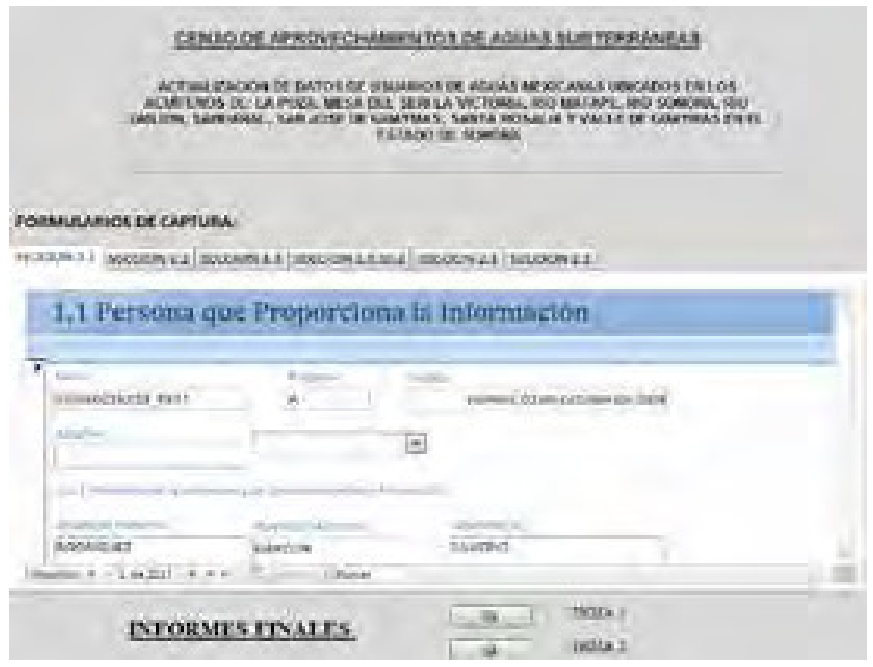

Figura 4.1. Vista de Entrada de la Base de Datos en Access

Cabe señalar, que de la correcta estructuración de las tablas que integran la base de dato depende la eficacia de la misma. Por tanto, uno de los puntos más importantes a considerar en el diseño de la base de datos, es la definición de tablas y las relaciones entre ellas. 
El primer paso consistió en realizar un análisis del problema a resolver para poder construir un diseño inicial de la base de datos que proyecte la magnitud de este. Es importante mencionar que debe tenerse un punto de vista general de las estructuras que almacenan los datos en el sistema. Finalmente, se construyeron los mecanismos de manipulación de dichas estructuras, como son, los formularios y objetos que facilitan el manejo de la base de datos.

En el diseño de cada tabla, deben determinarse los campos que contienen y definir las relaciones entre ellos. Esto no coacciona el hecho de que la tabla pueda modificarse en cualquier momento, sin embargo, esta acción puede conllevar la pérdida de la información previamente almacenada.

El correcto diseño de la base de datos garantiza que las operaciones que se realicen sean ejecutadas de manera precisa y eficaz.

\subsection{Propuesta de Software para el Desarrollo y Visualización}

El software seleccionado para el desarrollo del SIG fue ARCGIS, porque cumple con las características y requerimientos necesarios para llevar a cabo el proyecto.

ArcGIS es un completo sistema de información que permite crear, analizar, almacenar y difundir datos, modelos, mapas y globos en $3 \mathrm{D}$, poniéndolos a disposición de todos los usuarios según las necesidades de la organización.

Como sistema de información, ArcGIS es accesible desde clientes desktop, navegadores web, y terminales móviles que se conectan a servidores departamentales, corporativos o arquitecturas de computación en la nube. ArcGIS proporciona herramientas que permite crear aplicaciones personalizables.

\subsection{Captura de las encuestas en el SIG}

La información extraída de las encuestas almacenadas en Microsoft Access se captura en la SIG, en este, los usuarios son capaces de visualizar, crear, manipular y gestionar la información geográfica, estos corresponden a los aprovechamientos, direcciones, posiciones en terreno, áreas urbanas y rurales; regiones y cualquier tipo de ubicaciones en terrenos determinados. Esta información es trabajada de manera sistémica, lo que representa una diferencia sustancial a lo relacionado al trabajo con información planos y mapas, permitiéndonos explorar, ver y analizar los datos según parámetros, relaciones y tendencias que presenta nuestra información, teniendo como resultado nuevas capas de información, mapas y nuevas bases de datos. La figura 4.2 muestra cómo es que la información es ingresada al SIG, así como también el avance realizado por las brigadas en el barrido de campo del acuífero La Poza se muestra en la figura 4.3. Algunas funciones con las que se cuenta son: construir los mapas dinámicos e inteligentes que permiten a visualizar patrones, tendencias y singularidades en los datos. También, se incluye formas fáciles de levantar mapas, formatos predefinidos de mapas y una librería de elementos extensa, que permiten elaborar mapas de calidad rápidos y como el usuario desee. Los mapas terminados se pueden guardar, imprimir, exportary ubicar en otros documentos o usos. La SIG también permite visualizar datos como cartas, informes, con volumen, con gráficos e imágenes; teniendo la posibilidad de editarlos expeditamente.

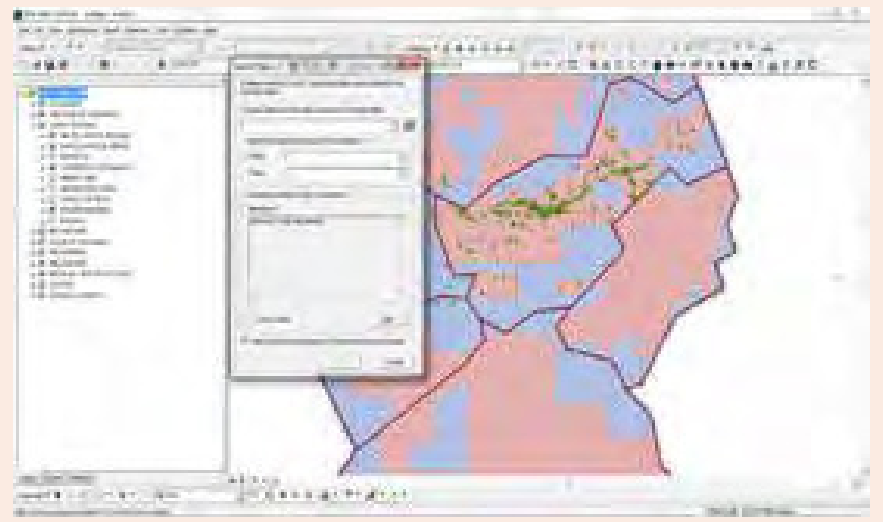

Figura 4.2. Interface para agregar información al sistema

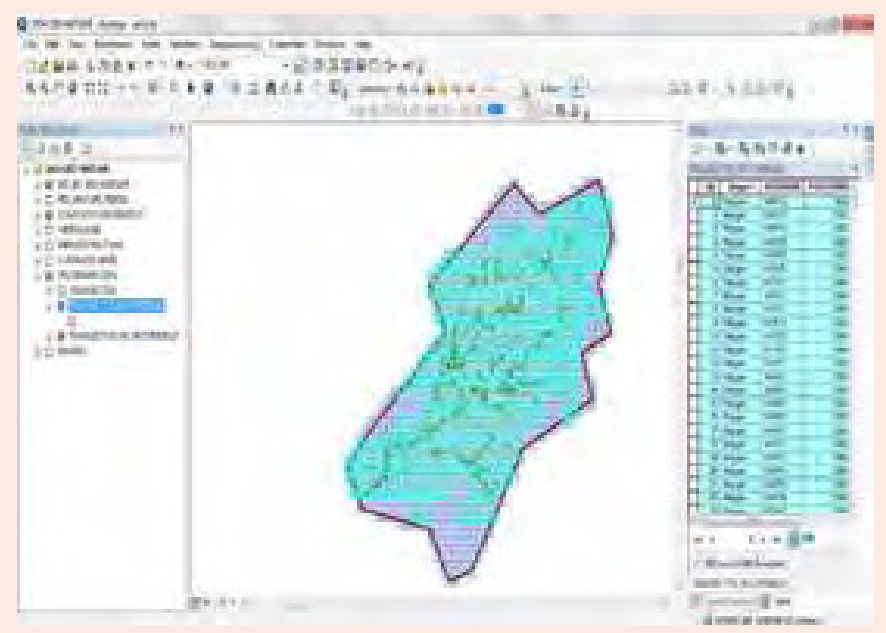

Figura 4.3. Avance realizado por las brigadas en el barrido de campo del acuífero de Rio Matape

\section{CONCLUSIONES}

Este proyecto define una metodología para realizar la actualización de los datos de usuarios de Aguas Mexicanas ubicados en los acuíferos de: LA POZA, MESA DEL SERI-LA VICTORIA, RIO MATAPE, RIO SONORA, RIO ZANJON, SAHUARAL, SAN JOSE DE GUAYMAS, SANTA ROSALIA Y VALLE DE GUAYMAS en el Estado de Sonora, mediante un Sistema de Información Geográfico, permite contar con un inventario actualizado, confiable y preciso 
de todos los aprovechamientos de aguas nacionales subterráneas en dichos Acuíferos, así como sus bienes públicos inherentes; por medio del cual se pueden llevar a cabo actos de autoridad en la materia con mayor confiabilidad y eficacia por conducto de la Subdirección General de Administración del Agua en cumplimiento de sus funciones y atribuciones legales.

Dicha actualización de datos en campo permite caracterizar a los usuarios del agua que cuentan con título de concesión, a los que no cuentan con título de concesión, a los que tienen más de un aprovechamiento por título y a los que no se encuentran en campo y cuentan con título de concesión.

Finalmente, el presente proyecto permite corregir los errores de localización geográfica que presenta el REPDA; así mismo, cuenta con una base de datos geoespacial por tipo de aprovechamiento debidamente requisitada en campo y asociada a la localización geográfica que le corresponda, para lo cual se utiliza el Sistema de Información Geográfica (SIG) denominado ARCGIS y sus respectivas extensiones.

\section{BIBLIOGRAFIA}

[1] Navarro José., Collado, Julián. (2009), “Practicas de SIG con ArcGIS", Editorial Universidad Politécnica de Valencia, Vol. 1, pp. 9-21.

[2] Moreno Jimenez, Antonio. (2008), "Sistemas y análisis de la información geográfica: manual de autoaprendizaje con ArcGIS", Editorial Alfaomega, Vol. 2, pp. 233-245.

[3] Wang Fanfang., Yang Han. (2009), "Study of the cultivated land and its salinization in the delta of Weigan River based on RS and GIS", Bingchuan Dongtu = Journal of Glaciology and Geocryology, Vol. 31 No. 4, pp. 672-678.

[4] Kumar G. Satish (2011), "Delineation of ground water potential zones using remote sensing and GIS in and around Holenarasipur schist belt, Karnataka, India.", Recent developments in ground water research in India, Vol. 26 No. 2, pp. 147-150.

[5] Varade Abhay M., Wadwekar Madhura, Kolte Dilip, Dongre K. P., Khare, Y. D. (2011), "Identification of ground water potential zones in and around Nagpur City, Maharashtra using remote sensing and GIS.", Gondwana Geological Magazine, Vol. 26 No. 2, pp. 129-138.

[6] D’Ercole Cecilia., Knox-Robinson., Carl M. (1997), “Application of a geographic information system for assessing MVT mineralisation potential of the southeastern Lennard Shelf, Western Australia.", Proceedings of the Biennial SGA Society for Geology Applied to Mineral Deposits Meeting, Vol. 4, pp. 855-857.

[7] Chenini Ismail., Ben Mammou Abdallah., El May Moufida. (2010), "Groundwater recharge zone mapping using GISbased multi-criteria Albacete Spain analysis; a case study in central Tunisia (Maknassy Basin)", Water Resources Management, Vol. 24 No. 5, pp. 931-939.

[8] Castano Santiago., Sanz David., Gomez-Alday Juan J. (2010). "Methodology for quantifying groundwater abstractions for agriculture via remote sensing and GIS.", Water Resources Management., Vol. 24, No. 4, 795-814.

[9] Kim Sung-Min., Choi Yosoon., Suh Jangwon., Oh Sungchan., Park Hyeong-Dong., Yoon Suk-Ho. (2012) "ArcMine; a GIS extension to support mine reclamation planning", Computers \& Geosciences, Vol. 46, pp. 84 - 95.

[10] McHugh Michael., Brand Cortney., Crawford Jim. (2002) “GIS- based quantification of groundwater availability for City of Aurora", American Water Resources Association Technical Publication Series, Vol. 2, No. 2, pp. 341-346.

[11] Rindahl, Bruce. (2002) "Linking consumptive use, surface water, and ground water models to create a decision support system; the GIS component.", American Water Resources Association Technical Publication Series, Vol. 2, No. 2, pp. 317-322.

[12] Hall Grahame., Cratchley Roger., Johnson, Sarah. (2006) "The use of SAGA-GIS in an integrated meteorological/ hydrological model for the Mawddach River catchment, north Wales.Novatica,", Goettinger Geographische Abhandlungen, Vol. 115, pp. 53-60.

[13] Levine Norman S., Clancy Kathleen E., Paz Emma L., Kaufman Charlie C. (2011) "Understanding local impacts of sea level rise; a GIS-based analysis in Charleston, SC.", Abstracts with Programs Geological Society of America, Vol. 43, No. 2, pp. 25-26.

[14] Ketchum Winn A. (2011) "Using GIS to analyze spatial distribution and taphonomic processes of the extinct tapir Tapirus polkensis at the Gray Fossil Site, Gray, Tennessee.", Abstracts with Programs Geological Society of America, Vol. 43, No. 2, pp. 25.

[15] Van Hoesen John G., Orndorff Richard L. (2011) "The morphology and spatial distribution of late Quaternary periglacial landforms, Snake Range, Nevada; a GISbased approach to prioritizing field sites.", Journal of the ArizonaNevada Academy of Science, Vol. 43, No. 1, pp. 48-60.

[16] Singh Vivek Kumar., Reddy D. V. R., Jayaswal Abhishek. (2008) "A study on information extraction and assessment of spatial distribution of overburden dumps in a coal mining area using remote sensing and GIS techniques.", International Journal of Earth Sciences and Engineering, Vol. 4, No. 3, pp. 98-101.

[17] Chenini Ismail., Ben Mammou Abdallah. (2010). "Ground water recharge study in arid region; an approach using GIS techniques and numerical modeling.", Computers \& Geosciences, Vol. 36, No. 6, pp. 801-817.

[18] Nuno Nicholas C., Davis Richard B., Steltenpohl Mark. Fousek Bob., West Randy. (2010). "GIS applications by industry of geologic and other pertinent data related to aggregate exploration and mining.,", Abstracts with Programs Geological Society of America, Vol. 42, No. 5, pp. 605.

[19] Kerski Joseph., Agrios Bronwyn., Johnson Ann., Lee, Angela. (2009). "New tools for investigating geoscience using GIS." Abstracts with Programs Geological Society of America, Vol. 41, No. 7, pp. 317.

[20] Russell Gail S. (2009). "Using Google Earth and GIS to facilitate access to geological information in state and local geological society guidebooks; northern Alabama Piedmont, 1973 to 2008.", Abstracts with Programs Geological Society of America., Vol. 41, No. 7, pp. 288-289.

[21] Futrell Mike. (2009) “Terrain modeling and GIS techniques for cave expeditions in China.", Proceedings of the International Congress of Speleology, Vol. 3, pp. 2078-2082.

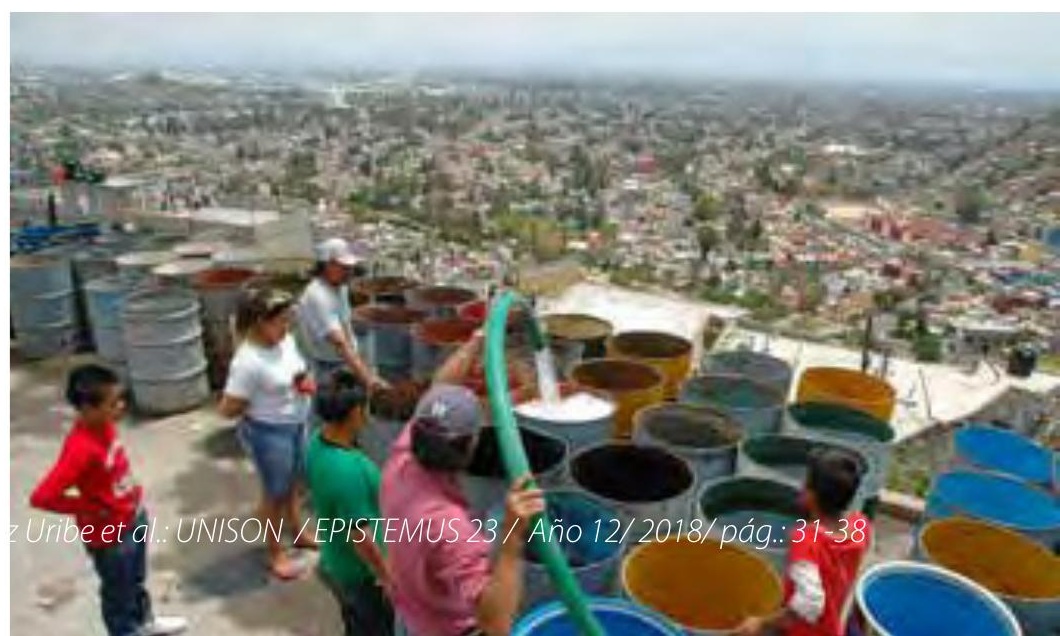

